\title{
Identification in Closed Loop: Some aspects on Direct and Indirect Approaches.
}

\author{
Lennart Ljung \\ Department of Electrical Engineering \\ Linköping University, S-581 83 Linköping, Sweden \\ WWW: http://www.control.isy.liu.se \\ Email: ljung@isy.liu.se
}

3 March 1999

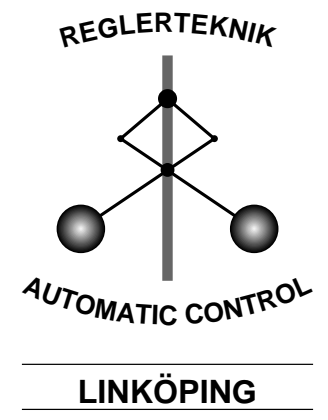

Report no.: LiTH-ISY-R-2101

For the IFAC Symposium on System Identification, Fukuoka, Japan 1997

Technical reports from the Automatic Control group in Linköping are available by anonymous ftp at the address ftp. control. isy. liu.se. This report is contained in the compressed postscript file 2101.ps.Z. 


\title{
IDENTIFICATION IN CLOSED LOOP: SOME ASPECTS ON DIRECT AND INDIRECT APPROACHES.
}

\author{
Lennart Ljung \\ Dept. of Electrical Engineering, Linköping University \\ S-581 83 Linköping, Sweden, \\ E-mail: ljung@isy.liu.se
}

\begin{abstract}
Identification for Control" has drawn significant interest the past few years. The objective is to achieve a model that is suited for robust control design. Thus one has to tailor the experiment and preprocessing of data so that the model is reliable in regions where the design process does not tolerate significant uncertainties. The use of closed loop experiments has been a prominent feature in these approaches, in particular various ways of handling the so-called indirect approach.

In this contribution we study a number of recently suggested methods and show how they correspond to different parameterizations. We also show how direct and indirect identification methods for closed loop data are linked together via the noise model. Some new results for bias distribution in closed loop experiments will also be presented.
\end{abstract}

Keywords: Identification, Closed-loop, Identifiability, Accuracy

\section{INTRODUCTION}

It is sometimes necessary to perform the identification experiment under output feedback (i.e., in closed loop). The reason may be that the plant is unstable, or that it has to be controlled for production economic or safety reasons, or that it contains inherent feedback mechanisms.

In this paper we shall review problems and possibilities with identification data from closed loop operation. A focus will be to interpret newly suggested methods and parameterizations.

In many cases we will not need to know the feedback mechanism, but for some of the analytic treatment we shall work with the following linear output feedback setup: The true system is

$y(t)=G_{0}(q) u(t)+v(t)=G_{0}(q) u(t)+H_{0}(q) e(t)$

Here $\{e(t)\}$ is white noise with variance $\lambda_{0}$. The

\footnotetext{
1 This work was supported in part by the Swedish Research Council for Engineering Sciences (TFR), which is gratefully acknowledged.
}

regulator is

$$
u(t)=r(t)-F_{y}(q) y(t)
$$

Here $\{r(t)\}$ is a reference signal (filtered version of a setpoint, or any other external signal $)$ that is independent of the noise $\{e(t)\}$. The model is

$$
y(t)=G(q, \theta) u(t)+H(q, \theta) e(t)
$$

We also assume that the closed loop is well defined in the sense that

$$
\begin{aligned}
& \text { EitherF } F_{y}(q) \text { or both } G(q, \theta) \text { and } G_{0}(q) \\
& \text { contain a delay }
\end{aligned}
$$

The closed loop system is stable

The closed loop equations become

$$
y(t)=G_{0}(q) S_{0}(q) r(t)+S_{0}(q) v(t)
$$

where $S_{0}(q)$ is the sensitivity function,

$$
S_{0}(q)=\frac{1}{1+F_{y}(q) G_{0}(q)}
$$

In the sequel we shall omit arguments $q, e^{i \omega}$ and $t$ whenever there is no risk of confusion. 
The input can be written

$$
u=S_{0} r-F_{y} S_{0} v
$$

The input spectrum is

$$
\Phi_{u}(\omega)=\left|S_{0}\right|^{2} \Phi_{r}(\omega)+\left|F_{y}\right|^{2}\left|S_{0}\right|^{2} \Phi_{v}(\omega)
$$

Here $\Phi_{r}$ and $\Phi_{v}$ are the spectra of the reference signal and the noise, respectively. We shall use the notation

$$
\begin{aligned}
& \Phi_{u}^{r}=\left|S_{0}\right|^{2} \Phi_{r} \\
& \Phi_{u}^{v}=\left|F_{y}\right|^{2}\left|S_{o}\right|^{2} \Phi_{v}
\end{aligned}
$$

to show the two components of the input spectrum, originating from the reference signal and the noise respectively.

\section{BIAS DISTRIBUTION}

We shall now characterize in what sense the model approximates the true system, when it cannot be exactly described within the model class. This will be a complement to the open loop discussion in Section 8.5 of (Ljung, 1987)

We have the prediction errors

$$
\begin{aligned}
\varepsilon & =\frac{1}{\mathrm{H}}(\mathrm{y}-\mathrm{Gu})=\frac{1}{\mathrm{H}}\left\{\left(\mathrm{G}_{0}-\mathrm{G}\right) \mathrm{u}+\mathrm{H}_{0} e\right\} \\
& =\frac{1}{\mathrm{H}}(\tilde{\mathrm{G}} \mathrm{u})+\left(\frac{\mathrm{H}_{0}}{\mathrm{H}}-1\right) e+e \\
& =\frac{1}{\mathrm{H}}(\tilde{\mathrm{G}} \mathrm{u}+\tilde{\mathrm{H}} e)+e
\end{aligned}
$$

Here

$$
\begin{aligned}
\tilde{\mathrm{G}} & =\mathrm{G}_{0}-\mathrm{G} \\
\tilde{\mathrm{H}} & =\mathrm{H}_{0}-\mathrm{H}
\end{aligned}
$$

Insert (8) for $\mathrm{u}$,

$$
\varepsilon=\frac{1}{\mathrm{H}}\left(\tilde{\mathrm{G}}\left(\mathrm{S}_{0} r-\mathrm{F}_{\mathrm{y}} \mathrm{S}_{0} \mathrm{H}_{0} e\right)+\tilde{\mathrm{H}} e\right)+e
$$

Under assumption (4), $\tilde{\mathrm{G}}(\mathrm{q}) \mathrm{F}_{\mathrm{y}}(\mathrm{q})$ contains a delay, as well as $\tilde{H}$ (since both $\mathrm{H}$ and $\mathrm{H}_{0}$ are monic). Therefore the last term of (14) is independent of the rest. The spectral density of $\varepsilon$ then becomes (overbar denotes complex conjugate)

$$
\begin{aligned}
\Phi_{\varepsilon} & =\frac{1}{|\mathrm{H}|^{2}}\left[\Phi_{u}|\tilde{\mathrm{G}}|^{2}-2 \operatorname{Re} \overline{\tilde{H}} \tilde{\mathrm{G}} \mathrm{F}_{y} S_{0} H_{0} \lambda_{0}+|\tilde{\mathrm{H}}|^{2} \lambda_{0}\right] \\
& +\lambda_{0}=\frac{\Phi_{u}}{|\mathrm{H}|^{2}}\left|\tilde{\mathrm{G}}-\frac{\overline{\mathrm{F}_{y} S_{0} \mathrm{H}_{0}}}{\Phi_{\mathrm{u}}} \lambda_{0}\right|^{2}+ \\
& +\frac{\lambda_{0}|\tilde{\mathrm{H}}|^{2}}{|\mathrm{H}|^{2}}\left[1-\frac{\Phi_{u}^{v}}{\Phi_{u}}\right]+\lambda_{0}
\end{aligned}
$$

Let us introduce the notation (B for "bias")

$$
\mathrm{B}=\frac{\overline{\mathrm{F}_{\mathrm{y}} \mathrm{S}_{0} \mathrm{H}_{0}} \tilde{\mathrm{H}} \lambda_{0}}{\Phi_{\mathrm{u}}}
$$

Note that

$$
|\mathrm{B}|^{2}=\frac{\lambda_{0}}{\Phi_{u}} \cdot \frac{\Phi_{u}^{v}}{\Phi_{u}} \cdot|\tilde{H}|^{2}
$$

The limiting model will minimize the integral of $\Phi_{\varepsilon}$, according to standard prediction error identification theory. We see that if $F_{y}=0$ (open loop operation) we have $B=0$ and $\Phi_{u}^{v}=0$ and we re-obtain expressions that are equivalent to the expressions in Section 8.5 in (Ljung, 1987).

Let us now focus on the case with a fixed noise model $\mathrm{H}(\mathrm{q}, \theta)=\mathrm{H}_{*}(\mathrm{q})$. This case can be extended to the case of independently parameterized $G$ and $\mathrm{H}$. Recall that any prefiltering of the data or prediction errors is equivalent to changing the noise model. The expressions below therefore contain the case of arbitrary prefiltering. For a fixed noise model, only the first term of (15) matters in the minimization, and we find that the limiting model is obtained as

$$
\mathrm{G}_{*}=\arg \min _{\mathrm{G}} \int_{-\pi}^{\pi}\left|\mathrm{G}_{0}-\mathrm{G}-\mathrm{B}\right|^{2} \frac{\Phi_{u}(\omega)}{\left|\mathrm{H}_{*}\right|^{2}} \mathrm{~d} \omega(18)
$$

This is identical to the open loop expression, except for the bias term B. Within the chosen model class, the model $G$ will approximate the biassed transfer function $G_{0}-B$ as well as possible according the the weighted frequency domain function above. The weighting function $\Phi_{u} /|\mathrm{H}|^{2}$ is the same as in the open loop case. The major difference is thus that an erroneous noise model (or unsuitable prefiltering) may cause the model to approximate a biassed transfer function.

Let us comment the bias function B. First, note that while $G$ (in the fixed noise model case) is constrained to be causal and stable, the term B need not be so. Therefore B can be replaced by its stable, causal component (the "Wiener part") without any changes in the discussion. Next, from (17) we see that the bias-inclination will be small in frequency ranges where either (or all) of the following holds

- The noise model is good ( $\tilde{\mathrm{H}}$ is small)

- The feedback contribution to the input spectrum $\left(\Phi_{u}^{v} / \Phi_{u}\right)$ is small

- The signal to noise ratio is $\operatorname{good}\left(\lambda_{0} / \Phi_{u}\right.$ is small)

In particular, it follows that if a reasonably flexible, independently parameterized noise model is used, then the bias-inclination of the G-estimate can be negligible.

\section{VARIANCE AND INFORMATION CONTENTS IN CLOSED LOOP DATA}

Let us now consider the asymptotic variance of the estimated transfer function $\hat{\mathrm{G}}_{\mathrm{N}}$ using the Asymptotic Black-Box theory of Section 9.4 in (Ljung, 1987). Note that the basic result

$$
\operatorname{Cov}\left[\begin{array}{l}
\hat{G}_{N} \\
\hat{H}_{N}
\end{array}\right] \approx \frac{n}{N} \Phi_{v}(\omega)\left[\begin{array}{cc}
\Phi_{u}(\omega) & \Phi_{u e}(\omega) \\
\Phi_{e u}(\omega) & \lambda_{0}
\end{array}\right]
$$

applies also to the closed loop case. Here $n$ is the model order, $\mathrm{N}$ the number of data, $\Phi_{v}$ the 
spectrum of $v(t)=H_{0}(q) e(t)$, and $\Phi_{u e}(\omega)$ the cross spectrum between input $u$ and noise source e. From this general expression we can directly solve for the upper left element:

$$
\operatorname{Cov} \hat{G}_{N}=\frac{n}{N} \Phi_{v}(\omega) \cdot \frac{\lambda_{0}}{\lambda_{0} \Phi_{u}(\omega)-\left|\Phi_{u e}(\omega)\right|^{2}}
$$

From (9) we easily find that

$$
\begin{aligned}
\lambda_{0} \Phi_{u} & -\left|\Phi_{u e}\right|^{2}=\lambda_{0}\left[\left|S_{0}\right|^{2} \Phi_{r}+\right. \\
& \left.+\left|F_{y}\right|^{2}\left|S_{0}\right|^{2}\left|H_{0}\right|^{2} \lambda_{0}-\left|F_{y}\right|^{2}\left|S_{0}\right|^{2}\left|H_{0}\right|^{2} \lambda_{0}\right]
\end{aligned}
$$

SO

$$
\operatorname{Cov} \hat{G}_{N}=\frac{n}{N} \frac{\Phi_{v}(\omega)}{\left|S_{0}\right|^{2} \Phi_{r}(\omega)}=\frac{n}{N} \frac{\Phi_{v}(\omega)}{\Phi_{u}^{r}(\omega)}
$$

The denominator of (21) is the spectrum of that part of the input that originates form the reference signal $r$. The open loop expression has the total input spectrum here.

The expression (21) - which also is the asymptotic Cramer-Rao lower limit - tells us precisely "the value of information" of closed loop experiments. It is the noise-to-signal ratio (where "signal" is what derives from the injected reference) that determines how well the open loop transfer function can be estimated. From this perspective, that part of the input that originates from the feed has no information value when estimating $\mathrm{G}$.

The expression (21) also clearly points to the basic problem in closed loop identification: The purpose of feedback is to make the sensitivity function small, especially at frequencies with disturbances and poor system knowledge. Feedback will thus worsen the measured data's information about the system at these frequencies.

Note, though, that the "basic problem" is a practical and not a fundamental one: There are no difficulties, per se, in the closed loop data, it is just that in practical use, the information contents is less. We could on purpose make closed loop experiments with good information contents (but poor control performance).

Note that the output spectrum is, according to $(6)$,

$$
\Phi_{y}=\left|G_{0}\right|^{2} \Phi_{u}^{r}+\left|S_{0}\right|^{2} \Phi_{v}
$$

The corresponding spectrum in open loop operation would be

$$
\Phi_{\mathrm{y}}^{\text {open }}=\left|\mathrm{G}_{\mathrm{o}}\right|^{2} \Phi_{\mathrm{u}}+\Phi_{v}
$$

This shows that it may still be desirable to perform a closed loop experiment: If we have large disturbances at certain frequencies we can reduce the output spectrum by $\left(1-\left|S_{0}\right|^{2}\right) \Phi_{v}$ and still get the same variance for $\widehat{\mathrm{G}}_{\mathrm{N}}$ according to (21).

\section{APPROACHES TO CLOSED LOOP IDENTIFICATION}

A directly applied prediction error method - applied as if any feedback did not exist - will work well and give optimal accuracy if the true system can be described within the chosen model structure (both regarding the noise model and the dynamics model). Nevertheless, due to the pitfalls in closed loop identification, several alternative methods have been suggested. One may distinguish between methods that

(1) Assume no knowledge about the nature of the feedback mechanism, and do not use $r$ even if known.

(2) Assume the regulator and the signal $r$ to be known (and typically of the linear form (2)

(3) Assume the regulator to be unknown, but of a certain structure (like (2).

If the regulator indeed has the form (2), there is no major difference between (1), (2) and (3): This noise-free relationship can be exactly determined based on a fairly short data record, and then also $r$ carries no further information about the system, if $u$ is measured. The problem in industrial practice is rather that no regulator has this simple, linear form: Various delimiters, anti-windup functions and other non-linearities will have the input deviate from (2), even if the regulator parameters (e.g. PID-coefficients) are known. This strongly favors approaches that do not assume linear regulators. The methods correspondingly fall into the following main groups (see Gustavsson et al (1977)):

(1) The Direct Approach: Apply the basic prediction error method (7.12) in a straightforward manner: use the output $y$ of the process and the input $u$ in the same way as for open loop operation, ignoring any possible feedback, and not using the reference signal $r$.

(2) The Indirect Approach: Identify the closed loop system from reference input $r$ to output $y$, and retrieve from that the open loop system, making use of the known regulator.

(3) The Joint Input-Output Approach: Consider $y$ and $u$ as outputs of a system driven by $r$ (if measured) and noise.

We shall discuss the two first methods in the following subsections.

\section{Direct Identification}

The Direct Identification approach should be seen as the natural approach to closed loop data analysis. The main reasons for this are

- It works regardless of the complexity of the regulator, and requires no knowledge about the character of the feedback.

- No special algorithms and software are required.

- Consistency and and optimal accuracy is obtained if the model structure contains the true system (including the noise properties).

There are two drawbacks with the direct approach: One is that we will need good noise mod- 
els. In open loop operation we can use output error models (and other models with fixed or independently parameterized noise models) to obtain consistent estimates (but not of optimal accuracy) of $\mathrm{G}$ even when the noise model $\mathrm{H}$ is insufficient. See Theorem 8.4 in (Ljung, 1987).

The second drawback is a consequence of this and appears when a simple model is sought that should approximate the system dynamics in a prespecified frequency norm. In open loop we can do so with the output error method and a fixed prefilter/noise model that matches the specifications. For closed loop data a prefilter/noise model that deviates considerably from the true noise characteristics will introduce bias, according to (18).

The natural solution to this would be to first build a higher order model using the direct approach, with small bias, and then reduce this model to lower order with the proper frequency weighting.

Another case that shows the necessity of good noise models concerns unstable systems. For closed loop data, the true system to be identified could very well be unstable, although the closed loop system naturally is stable. The prediction error methods require the predictor to be stable. This means that any unstable poles of $G$ must be shared by $\mathrm{H}$, like in ARX, ARMAX and statespace models. Output error models cannot be used for this case. Just as in the open loop case, models with common parameters between $\mathrm{G}$ and $\mathrm{H}$ require a consistent noise model for the G-estimate to be consistent.

\section{Indirect Identification}

The closed loop system under (2) is

$$
\begin{aligned}
y(t) & =G_{c l}(q) r(t)+v_{c l}(t)= \\
& =\frac{G_{0}(q)}{1+F_{y}(q) G_{0}(q)} r(t)+\frac{1}{1+F_{y}(q) G_{0}(q)} v(t)
\end{aligned}
$$

The indirect approach means that $\mathrm{G}_{\mathrm{cl}}$ is estimated from measured $y$ and $r$, giving $\widehat{G}_{\mathrm{cl}}$, and then the open loop transfer function estimate $\widehat{G}$ is retrieved from the equation

$$
\widehat{\mathrm{G}}_{\mathrm{cl}}=\frac{\hat{\mathrm{G}}}{1+\hat{\mathrm{G} F}}
$$

An advantage with the indirect approach is that any identification method can be applied to (23) to estimate $\widehat{\mathrm{G}}_{\mathrm{cl}}$, since this is an open loop problem. Therefore methods like spectral analysis, instrumental variables, and subspace methods, that may have problems with closed loop data, also can be applied.

For methods, like the prediction error method, that allow arbitrary parameterizations $\mathrm{G}_{\mathrm{cl}}(\mathrm{q}, \theta)$ it is natural to let the parameters $\theta$ relate to properties of the open loop system G, so that

$$
\mathrm{G}_{\mathrm{cl}}(\mathrm{q}, \theta)=\frac{\mathrm{G}(\mathrm{q}, \theta)}{1+\mathrm{F}_{\mathrm{y}}(\mathrm{q}) \mathrm{G}(\mathrm{q}, \theta)}
$$

That will make the task to retrieve the open loop system from the closed loop one more immediate.

We shall now assume that $G_{c l}$ is estimated using a prediction error method with a fixed noise model/prefilter $\mathrm{H}_{*}$ :

$$
y(t)=G_{c l}(q, \theta) r(t)+H_{*}(q) e(t)
$$

The parameterization can be arbitrary, and we shall comment on it below. It is quite important to realize that as long as the parameterization describes the same set of $\mathrm{G}$, the resulting transfer function $\hat{\mathrm{G}}\left(\mathrm{q}, \hat{\theta}_{\mathrm{N}}\right)$ will be the same, regardless of the parameterizations. The choice of parameterization may thus be important for numerical and algebraic issues, but it does not affect the statistical properties of the estimated transfer function.

Let us now discuss bias and variance aspects of $\hat{G}$ estimated from (26) and (25). We start with the variance. According to the open loop result, the asymptotic variance of $\widehat{\mathrm{G}}_{\mathrm{cl}, \mathrm{N}}$ will be

$$
\operatorname{Cov} \widehat{G}_{c l, N}=\frac{n}{N} \frac{\Phi_{v, c l}(\omega)}{\Phi_{r}(\omega)}=\frac{n}{N} \frac{\left|S_{0}\right|^{2} \Phi_{v}}{\Phi_{r}}
$$

regardless of the noise model $\mathrm{H}_{*}$. Here $\Phi_{v, \mathrm{cl}}$ is the spectrum of the additive noise $v_{\mathrm{cl}}$ in the closed loop system (23), which equals the open loop additive noise, filtered through the true sensitivity function. To transform this result to variance of the open loop transfer function, we use Gauss' approximation formula

$$
\operatorname{Cov} \hat{\mathrm{G}}=\frac{\mathrm{dG}}{\mathrm{dG}_{\mathrm{cl}}} \operatorname{Cov} \hat{\mathrm{G}}_{\mathrm{cl}} \frac{\mathrm{dG}}{\mathrm{dG}_{\mathrm{cl}}}{ }^{*}
$$

It is easy to verify that

$$
\frac{\mathrm{dG}}{\mathrm{dG}_{\mathrm{cl}}}=\frac{1}{\mathrm{~S}_{\mathrm{O}}^{2}}
$$

So

$$
\operatorname{Cov} \hat{\mathrm{G}}_{\mathrm{N}}=\frac{\mathrm{n}}{\mathrm{N}} \frac{\Phi_{v}}{\left|\mathrm{~S}_{0}\right|^{2} \Phi_{\mathrm{r}}}=\frac{\mathrm{n}}{\mathrm{N}} \frac{\Phi_{v}}{\Phi_{u}^{r}}
$$

which - not surprisingly - equals what the direct approach gives, (21). In fact, the following more general result can be proven, see Gustavsson et al (1976): Suppose that the closed loop system, including a noise model, is consistently estimated with a prediction error method. Let the open loop system be solved from (25). In case this is an overdetermined system of equations, solve for it in the least squares sense, using the estimated covariances as weights. Then the indirectly identified model has the same accuracy (covariance properties) as a directly identified model.

For the bias, we know that the limiting estimate $\theta^{*}$ is given by (we write $G_{\theta}$ as short for $G\left(e^{i \omega}, \theta\right)$ )

$$
\begin{aligned}
\theta^{*} & =\arg \min \int_{-\pi}^{\pi}\left|\frac{\mathrm{G}_{0}}{1+\mathrm{F}_{y} \mathrm{G}_{0}}-\frac{\mathrm{G}_{\theta}}{1+\mathrm{F}_{y} \mathrm{G}_{\theta}}\right|^{2} \frac{\Phi_{\mathrm{r}}}{\left|\mathrm{H}_{*}\right|^{2}} \mathrm{~d} \omega \\
& =\arg \min \int_{-\pi}^{\pi}\left|\frac{\mathrm{G}_{0}-\mathrm{G}_{\theta}}{1+\mathrm{F}_{y} \mathrm{G}_{\theta}}\right|^{2} \frac{\left|\mathrm{S}_{0}\right|^{2} \Phi_{\mathrm{r}}}{\left|\mathrm{H}_{*}\right|^{2}} \mathrm{~d} \omega
\end{aligned}
$$


Now, this is no clear cut minimization of the distance $G_{0}-G$. The estimate $\theta^{*}$ will be a compromise between making $G$ close to $G_{0}$ and making $1 /\left(1+F_{y} G\right)$ (the model sensitivity function) small. There will thus be a "bias-pull" towards transfer functions that give a small sensitivity for the given regulator, but unlike (18) it is not easy to quantify this bias component. However, if the true system can be represented within the model set, this will always be the minimizing model, so there is no bias in this case.

\section{SPECIAL PARAMETERIZATIONS FOR INDIRECT METHODS}

The above results are independent of how the closed loop system is parameterized. A nice and interesting parameterization for this indirect identification of closed loop systems has been suggested by (Hansen, 1989), (Hansen and an R. L. Kosut, 1989), (Schrama, 1991a) and (Schrama, 1991b). It is based on so-called YoulaKucera parameterization and works as follows for the SISO case:

Write the regulator $F_{y}=X / Y$ for some stable transfer functions (e.g. polynomials) $\mathrm{X}$ and $\mathrm{Y}$. Let $N$ and $D$ be any stable transfer functions, such that $\mathrm{XN}+\mathrm{YD}$ is stable and inversely stable. (This means that $G_{\text {nom }}=N / D$ is a system that would be stabilized by $F_{y}$.) Now, parameterize $G$ in terms of a stable transfer function $S_{\theta}$ as

$$
\mathrm{G}(\mathrm{q}, \theta)=\mathrm{G}_{\theta}=\frac{\mathrm{N}+\mathrm{YS} \mathrm{S}_{\theta}}{\mathrm{D}-X \mathrm{~S}_{\theta}}
$$

This set of models ranges over all systems that are stabilized by $F_{y}$ as $S_{\theta}$ ranges over the set of stable transfer functions. This is clearly a nice feature, since it is natural to look for a good model in precisely this set. However, order constraints on $\mathrm{G}_{\theta}$ do not correspond to simple constraints on $S_{\theta}$, which might be a disadvantage.

Simple manipulations now give that

$$
\mathrm{G}_{\mathrm{cl}}(\mathrm{q}, \theta)=\operatorname{LY}\left(\mathrm{N}+\mathrm{S}_{\theta} \mathrm{Y}\right)
$$

where $\mathrm{L}=1 /(\mathrm{YD}+\mathrm{NX})$, which was stable by construction. The estimation problem (26) now reads

$$
\begin{aligned}
y(t) & =L(q) N(q) Y(q) r(t)+ \\
& +S(q, \theta) L(q) Y^{2}(q) r(t)+H_{*}(q) e(t)
\end{aligned}
$$

Estimating $\theta$ from this is of course equivalent to estimating it from

$$
z(t)=S(q, \theta) x(t)+H_{*}(q) e(t)
$$

where

$$
\begin{aligned}
& z(t)=y(t)-L(q) N(q) Y(q) r(t) \\
& x(t)=L(q) Y^{2}(q) r(t)
\end{aligned}
$$

The formulation (33) with $S$ being any stable transfer function of a certain order is a standard open loop identification problem, but it is important to realize that it is a special parameterization of the general indirect identification approach.
Finally it should be remarked that the indirect approach is critically dependent on the knowledge of $F_{y}$. The closed loop system is consistently estimated, so any error in $F_{y}$ will directly lead to a corresponding error in $G$, when solved for from the closed loop system.

\section{PERIODIC REFERENCE SIGNALS AND TIME-INVARIANT REGULATORS}

A very useful property for closed loop experiments in connection with "identification-for-control" would be to have a method that allows fitting the model to the data in a fixed, model-independent and user defined frequency domain norm. This is possible for open loop data using prefiltering and an output error model/method. (Like in (18) with $\mathrm{B}=0$.) For closed loop using direct or indirect methods, we either get bias as in (18) or modeldependent norms as in (29).

For the case of periodic reference signals and timeinvariant regulators (McKelvey, 1996) has pointed out and analyzed such a method: For a periodic reference signal, the parts of $u$ and $y$ that originate from $r$ will be periodic after a transient. Now, average $y$ and $u$ over periods corresponding to the period of $r$. These averages will then converge to a correct, noise-free input-output relationship for the system over one period. Then use these averages as input and outputs in a direct output-error identification scheme, possibly with prefiltering. This gives a method with the desired properties.

Also the so called two-step method of (van den Hof and Schrama, 1993) (which we would classify as a joint input-output method) will allow - under certain assumptions - the user to fit the model to data in a known, and user-chosen, frequency domain norm.

\section{A FORMAL CONNECTION BETWEEN DIRECT AND INDIRECT METHODS}

The noise model $\mathrm{H}$ in a linear system model structure has often turned out to be a key to interpretation of different "methods". The distinction between the models/"methods" ARX, ARMAX, Output Error, Box-Jenkins, etc, is entirely explained by the choice of the noise model. Also the practically important feature of prefiltering is equivalent to changing the noise model. Even the choice between minimizing one- or $\mathrm{k}$-step prediction errors can be seen as a noise model issue. See, e.g. (Ljung, 1987), for all this.

Therefore it should not come as a surprise that also the distinction between the fundamental approaches of Direct and Indirect identification can be seen as a choice of noise model.

One important point of the prediction error approach is that the transfer functions $G$ and $H$ can be arbitrarily parameterized. Suppose that we have a closed loop system with known regulator 
$F_{y}$ as before. We parameterize $G$ as $G(g, \theta)$ and $\mathrm{H}$ as

$$
H(q, \theta)=H_{1}(q, \theta)\left(1+F_{y}(q) G(q, \theta)\right)
$$

We thus link the noise model to the dynamics model. There is nothing strange with that: So do ARX and ARMAX models. Note that this particular parameterization scales $\mathrm{H}_{1}$ with the inverse model sensitivity function.

Now, the predictor for

$$
y(t)=G(q, \theta)+H(q, \theta)
$$

is

$$
\begin{aligned}
\hat{y}(t \mid \theta) & =H^{-1}(q, \theta) G(q, \theta) u(t) \\
& +\left(1-H^{-1}(q, \theta)\right) y(t) \\
& =\frac{H_{1}^{-1}(q, \theta) G(q, \theta)}{1+F_{y}(q) G(q, \theta)}\left(r(t)-F_{y}(q) y(t)\right) \\
& +y(t)-H_{1}^{-1}(q, \theta) \frac{1}{1+F_{y}(q) G(q, \theta)} y(t) \\
& =H_{1}^{-1}(q, \theta) \frac{G(q, \theta)}{1+F_{y}(q) G(q, \theta)} r(t)+ \\
& +\left(1-H_{1}^{-1}(q, \theta)\right) y(t)
\end{aligned}
$$

Now, this is exactly the predictor also for the model of the closed loop systems

$$
y(t)=G_{c l}(q, \theta) r(t)+H_{1}(q, \theta) e(t)
$$

with the closed loop transfer function parameterized in terms of the open loop one, as in (25).

The indirect approach to estimate the system in terms of the closed loop model (37) is thus identical to the direct approach with the noise model (34). This is regardless of the parameterization of $\mathrm{G}$ and $\mathrm{H}_{1}$. Among other things, this shows that we can use any theory developed for the direct approach (allowing for feedback) to evaluate properties of the indirect approach.

\section{SUMMARIZING REMARKS}

We may summarize the basic issues on closed loop identification as follows:

- The basic problem with closed loop data is that it typically has less information about the open loop system - an important purpose of feedback is to make the closed loop system insensitive to changes in the open loop system.

- Prediction error methods, applied in a direct fashion, with a noise model that can describe the true noise properties still gives consistent estimates and optimal accuracy. No knowledge of the feedback is required. This should be regarded as a prime choice of methods.

- Several methods that give consistent estimates for open loop data may fail when applied in a direct way to closed loop identification. This includes spectral and correlation analysis, the instrumental variable method, the subspace methods and output error methods with incorrect noise model.
- If the regulator mechanism is correctly known, indirect identification can be applied. Its basic advantage is that the dynamics model G can be correctly estimated even without estimating any noise model.

We should finally add that we have not treated joint input-output methods in this exposé. These offer quite interesting possibilities. See (van den Hof and Schrama, 1995), (Gevers et al., 1997) and (Forsell and Ljung, 1997) for some recent discussions.

\section{REFERENCES}

Forsell, U. and L. Ljung (1997). Closed-loop identification revisited. Technical report. Dept of Electrical Engineering.

Gevers, M., L. Ljung and P. Van den Hof (1997). Asymptotic variance expressions for closedloop identification and their relevance in identification for control. In: Proc. IFAC Symposium on System Identification, SYSID'97. Fukuoka, Japan.

Hansen, F. R. (1989). A fractional representation approach to closed-loop system identification and experiment desing. PhD thesis. Stanford University. Stanford, CA, USA.

Hansen, F. R. and G. F. Franklin an R. L. Kosut (1989). Closed-loop identification via the fractional representation: Experiment design. In: Procceeding American Control Conference. pp. 386-391.

Ljung, L. (1987). System Identification - Theory for the User. Prentice-Hall. Englewood Cliffs, N.J.

McKelvey, T. (1996). Periodic excitation for identification of dynamic errors-in-variables systems operating in closed loop. In: Proc. 13th IFAC World Congress (J.J. Gertler, Jr J. B. Cruz and M. Peshkin, Eds.). Vol. J. San Francisco, CA. pp. 155-160. Paper no 3a-20-3.

Schrama, R. J. P. (1991a). Control-oriented approximate closed-loop identification via fractional representations. In: Proc. American Control Confernce. Boston, MA. pp. 719-720.

Schrama, R. J. P. (1991b). An open-loop solution to the approximate closed-loop identification problem. In: Proc. 9th IFAC Symposium on Identification and System Parameter Estimation. Budapest. pp. 1602-1607.

van den Hof, P. M. J. and R.J.P. Schrama (1993). An indirect method for transfer function estimation from closed loop data. Automatica 29(6), 1523-1527.

van den Hof, P. M. J. and R.J.P. Schrama (1995). Identification and control-closed loop issues. Automatica 31(12), 1751-1770. 